\title{
Model Interacting Particle Systems for Simulation and Macroscopic Description of Particulate Suspensions (DE-FG-02-03ER25567) FinAL REPORT
}

\author{
PI: Peter J. Mucha \\ Department of Mathematics \& Institute for Advanced Materials \\ University of North Carolina, Chapel Hill 27599-3250 \\ 919/843-2550, 919/962-9345(fax), mucha@unc.edu, http://www.unc.edu/ mucha
}

August 30, 2007

Period of Time Covered: September 1, 2003 to May 31, 2007.

Review of Proposed Goals: Suspensions of solid particles in liquids appear in numerous applications, from environmental settings like river silt, to industrial systems of solids transport and water treatment, and biological flows such as blood flow. Despite their importance, much remains unexplained about these complicated systems. Mucha's research aims to improve understanding of basic properties of suspensions through a program of simulating model interacting particle systems with critical evaluation of proposed continuum equations, in close collaboration with experimentalists.

Natural to this approach, the original proposal centered around collaboration with studies already conducted in various experimental groups. However, as was detailed in the 2004 progress report, following the first year of this award, a number of the questions from the original proposal were necessarily redirected towards other specific goals because of changes in the research programs of the proposed experimental collaborators. Nevertheless, the modified project goals and the results that followed from those goals maintain close alignment with the main themes of the original proposal, improving efficient simulation and macroscopic modeling of sedimenting and colloidal suspensions. In particular, the main investigations covered under this award have included

1. Sedimentation instabilities, including the sedimentation analogue of the Rayleigh-Taylor instability (for heavy, particle-laden fluid over lighter, clear fluid).

2. Ageing dynamics of colloidal suspensions at concentrations above the glass transition, using simplified interactions.

3. Stochastic reconstruction of velocity-field dependence for particle image velocimetry (PIV).

4. Stochastic modeling of the near-wall bias in "nano-PIV".

5. Distributed Lagrange multiplier simulation of the "internal splash" of a particle falling through a stable stratified interface.

6. Fundamental study of velocity fluctuations in sedimentation.

7. Parallelization of Mucha's "sort-and-sweep" sedimentation code.

Details about publications and collaborators are provided topic-by-topic in the sections below. 


\section{Sedimentation Instabilities}

- "Rayleigh-Taylor instabilities in sedimentation,"

S. Guda, S. Bukharina, and P. J. Mucha, in preparation.

- Swathi Guda, "Rayleigh-Taylor Instability in Sedimenting Suspensions," M.S. thesis, University of North Carolina at Chapel Hill, 2006.

- Svetlana Bukharina, M.S. project, Georgia Institute of Technology, 2005.

- Presentation of results at the annual APS Division of Fluid Dynamics meeting, by Svetlana Bukharina in 2004 and Mucha in 2007.

The application of main ideas in sedimentation instabilities has always been one of the primary themes of this project. In particular, there are interesting proposals [3] and experimental results (from A. E. Hosoi's group in Mechanical Engineering at MIT) regarding the fundamental Rayleigh-Taylor-like instability of heavy, particle-laden fluid over lighter, clear fluid. We have made substantial progress in further understanding this instability, at least in the context of dilute sedimentation accessible by Mucha's specialized particle dynamics simulations. Georgia Tech (GT) graduate student Svetlana Bukharina used these simulations to investigate critically the scaling of the dominant unstable wavenumber with system size, demonstrating a strong dependence on system size. Later, University of North Carolina at Chapel Hill (UNC) graduate student Swathi Guda extended this work further to extract the growth rate v. wavenumber dispersion relation of the instability from simulations, in excellent agreement with the high Rayleigh number limit of recent results about the instability between miscible fluids [4] (see Figure 1).
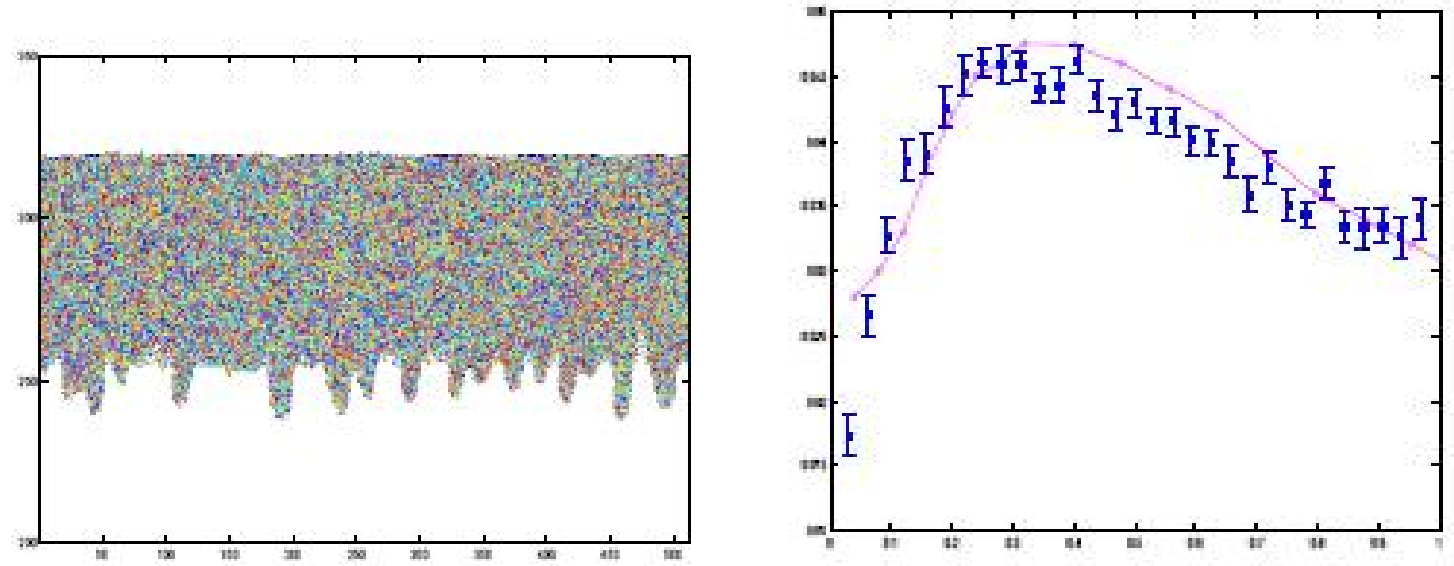

Figure 1: Rayleigh-Taylor instabilities in sedimentation. (a) A sample image from a simulation of the effect, showing the fingering of the lower unstable interface (the upper interface is stable). (b) Computationally-extracted growth rates v. wavenumber $k$, compared with the miscible RayleighTaylor results of Graf et al.

While these results are not particularly surprising a posteriori, this result completely undermines the use of immiscible surface-tension models (which have been proposed and used by others) to 
describe the clarified/particles interface, in that there is no microscopically-defined surface tension determining the dominant wavenumber; rather the dominant wavenumber is determined, at least in part, by the geometry and size of the container. Moreover, the study of the linear instability of the unstable interface does not appear to be at all influenced by fluctuation-induced effective diffusion (cf. the stable interface [5]), which can be physically understood in terms of the time scale of the linear instability being much faster than that of swirl turnover above which the effective diffusion model holds.

\section{Colloidal Ageing}

- Radleigh Santos, M.S. project, Georgia Institute of Technology, 2005.

Mucha remained actively involved during his GT appointment in regular discussions and directions to try to elucidate the colloidal ageing results in Eric Weeks' lab at Emory, by way of simulation of model particle interactions. Three graduate students worked on this problem off and on over the 2003-2005 years. First, Christel Hohenegger started on this project but then proceeded onto the nano-PIV problem (below), originally as a means of warming up to the proper study of the key effects of Brownian motion. Hwakil Kim then addressed this problem with some model numerical results during a semester supported under this award. Finally, Radleigh Santos, while not directly supported by the DOE (instead supported as a fellow under the GT School of Mathematics' NSF VIGRE award), did his master's project on this problem. Various different simplified model interactions were considered and computationally simulated, without finding a suitably simplified interaction which yielded the same qualitative behavior as the physical experiment that could be used for further theoretical study. With Mucha's departure from GT in Summer 2005, and because of the limited success of this specific project to that time, efforts were reassigned even further onto the study of the stochastic effects in nano-scale particle image velocimetry (nano-PIV), as described in the next two sections.

\section{Stochastic Reconstruction for PIV}

- "Statistical reconstruction of velocity profiles for nano-particle image velocimetry," C. Hohenegger and P. J. Mucha, to appear in SIAM Journal of Applied Mathematics.

- Christel Hohenegger, "Small scale stochastic dynamics for particle image velocimetry applications," Ph.D. thesis, Georgia Institute of Technology, 2006.

- Presentation of results at the annual APS Division of Fluid Dynamics meeting, by Christel Hohenegger in 2004 and by Mucha in 2005.

In trying to build our intuition for reduced order modeling of the hydrodynamic interactions between Brownian particles, as needed for example for further study of colloidal ageing, we started to consider and then became further involved with a specific experiment with simpler Brownian effects, the nanoscale particle image velocimetry (nano-PIV or nPIV) measurements in Minami Yoda's group (Mechanical Engineering, Georgia Tech) [7, 8], where the predominant interaction modeled is the hydrodynamics between a colloidal particle and the wall. Despite this simplicity, 


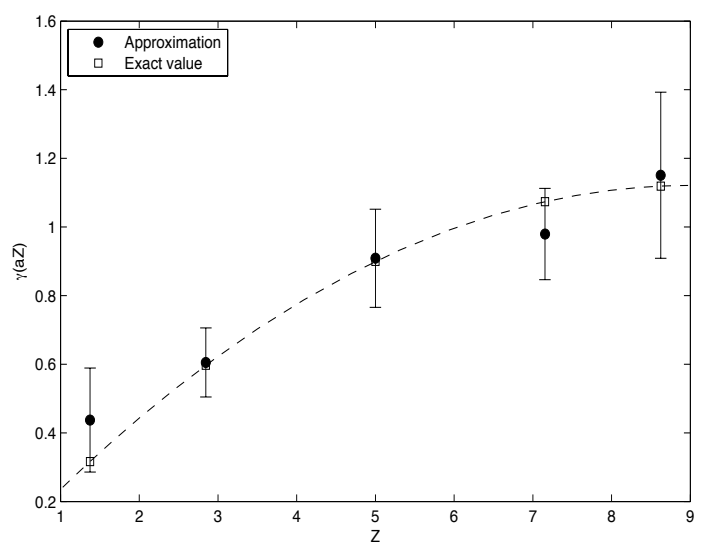

(a) Parabolic profile

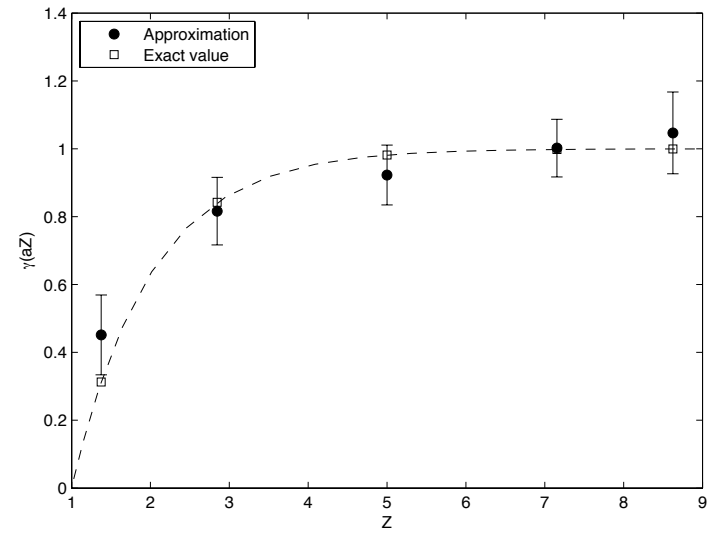

(b) Exponentially decaying profile

Figure 2: Stochastic reconstruction of sample velocity profiles [(a): parabolic, (b):exponentially decaying], with $M=5$ reconstructed values at different positions perpendicular to the PIV images (90\% confidence interval error bars).

high-order numerical solution of the resulting system of stochastic differential equations by Milstein methods requires the proper construction of numerically evaluated Ito integrals, because the system is not "diagonal" in the stochastic sense (the particle diffusivity in each component depends on only one of the position components). Using these calculations (developed largely by graduate student Christel Hohenegger, who was the primary benefactor of this award in terms of semesters of support), we have investigated limitations and advantages brought on by the strong Brownian effects at these small scales. In particular, given drifts and diffusivities that are correlated through the same coordinate, one can in principle use in-plane PIV to statistically extract drift dependence on the out-of-plane coordinate. Our simulations have successfully demonstrated this reconstruction (see, e.g., Figure 2) for PIV with ideal particle identification and for velocities obtained through proper cross-correlations of simulated images. We have also explored in detail the possibility of extending these "proof of concept" simulation results to real physical data obtained in the nano-PIV experiments, which led in large part to the result described in the next section.

\section{Diffusion-induced bias in near-wall velocimetry}

- "Diffusion-Induced Bias in Near-Wall Velocimetry," R. Sadr, C. Hohenegger, H. Li, P. J. Mucha and M. Yoda, Journal of Fluid Mechanics 577, 443-456 (2007).

- Christel Hohenegger, "Small scale stochastic dynamics for particle image velocimetry applications," Ph.D. thesis, GT, 2006.

Growing directly out of the collaboration that motivated our stochastic reconstruction efforts described above, Hohenegger and Mucha also provided fundamental mathematical and computational analysis to a bias problem in near-wall velocimetry at small scales that was initially identified by Minami Yoda's experimental group (as funded by the Air Force). The physical origin of the 
bias is that particles in the imaged region near the wall in the first exposure can diffuse beyond the imaged region away from the wall and back in time to be there in the second exposure. That is, the displacement of these particles between the two exposures used in the PIV measurement of velocity effectively averages spatially over the distribution of the matched particles' positions during the time between the exposures, which need not correspond to being centered in the imaged region as is typically assumed.

Following from the experimental observations, Hohenegger and Mucha studied the FokkerPlanck description for this diffusive process, including numerical solutions reproducing the experimental observations and the mathematical development of an empirical approximation for the average particle position sampled (the most important quantity for correcting this bias) as a function of physical parameters of the PIV imaging. This empirical approximation is being used by other groups in the analysis of their near-wall data (private communications, citations presumably coming soon). The paper presenting these results appeared in Journal of Fluid Mechanics earlier this year, acknowledging both the experimental support from the Air Force and the mathematical support from the DOE. Because of its relatively brief summation of important results, this is the paper selected to be separately sent to the program manager to be included as an appendix to the present report.

Additional mathematical questions have also been investigated beyond those results published in the manuscript listed above, particularly towards modeling the effect of finite exposure times (cf. the time between the exposures), which typically has the effect of biasing the measurement in the other direction (towards the wall). If these further mathematical investigations bear fruit, obviously the DOE will be further acknowledged in any follow-on publications.

\section{Simulation of internal splashes}

Consistent with the theme of instabilities and odd behaviors between solid objects and fluids, we have undertaken an as yet unfinished computational study of solid particles settling through fluids - this time passing through the stable diffuse interface between two fluids at different densities (light over heavy). Recent experiments at UNC [1] have uncovered an interesting "internal splash" behavior when a solid particle is dropped through a stable density interface in the fluid (say, at the interface between fresh and salt water). Abaid et al. have experimentally observed the entrainment of the lighter fluid into the heavier fluid giving rise to a plume that provides additional drag and can under some conditions reverse the motion of the falling particle. While they have a simple model for this behavior, its range of validity remains unknown. Meanwhile, the experiments are very time-consuming because of the difficulty of setting up the interface, while simulations of this behavior would seem to be exceptionally difficult. However, using a simulation code developed by Mucha and his collaborators for animation purposes [2], based significantly on the distributed Lagrange multipliers work of Patankar \& Joseph [6], we attempted numerical simulations of this phenomenon. This work has being carried out primarily by GT College of Computing Ph.D. students Chris Wojtan and Nipun Kwatra. Both 2-D and 3-D codes specially set up for studying this specific phenomena have been developed and thoroughly tested. Numerical exploration using that code will be an ongoing project for a future student. Clearly the DOE award will be cited in any future publications or presentations resulting from the use of this code. 


\section{Velocity fluctuations}

- "Velocity fluctuations of initially-stratified sedimenting spheres," S.-Y. Tee, P. J. Mucha, M. P. Brenner and D. A. Weitz, submitted.

- Presentation of results at the annual APS Division of Fluid Dynamics meeting, by Mucha in 2005.

In the last two years of this award, Mucha's research included further fundamental study of velocity fluctuations in sedimentation. The specific investigation of the submitted manuscript, of velocity fluctuations in the presence of an initially-imposed stratification of the particle concentration, started as part of an NSF award (DMS-0204309, 2002-2005), that award funding fundamental study of velocity fluctuations under different situations, including initial stratifications, propagation of the stable front (cf. the unstable front in the present award), bidisperse collections of particles, and fluidized beds. The end date of that NSF award in Summer 2005 arrived with the initiallystratified project unfinished. Relatively little additional time and financial support from the present award was used to finish that project, leading to a manuscript presently under review at Physics of Fluids which properly acknowledges both the NSF and the DOE.

\section{Parallelization of "sort-and-sweep" code}

The last result discussed here is purely computational: the parallelization of Mucha's previouslyexisting code for dilute-limit creeping flow simulations of sedimentation and other suspensions phenomena in bounded domains. These simulations were originally developed under an NSF Mathematical Sciences Postdoctoral Fellowship award (DMS-9902363, 1999-2002), and used extensively in Mucha's various investigations of sedimentation phenomena, including the Rayleigh-Taylor instabilities investigated under this award.

In the 2003 proposal for this award, a scheme for spatially partitioning the domain was sketched out. Specifically, the proposed parallelization scheme would have generalized directly from the "sort-and-sweep" nature of the original code, where the dilute-limit interactions between no-slip side walls are calculated in terms of field coefficients that can be efficiently evaluated to generate velocities, provided those evaluations occur sorted in the spatial coordinate perpendicular to the side walls. Unfortunately, despite many efforts to work through the details of such an algorithm, and in particular worrying about the resulting spatial heterogeneities of computational effort and the required message passing between spatial computational cells, a full algorithm utilizing these ideas remained incomplete. Additional consideration of this scheme included extensive discussions with the Star-P team at Interactive SuperComputing, but did not lead to a suitable algorithm.

Near the end of this award period, a simpler parallelization scheme became obvious; instead of partitioning spatially in the direction perpendicular to the walls, one can partition tasks in the 2-D wavevector space corresponding to the directions parallel to the side walls. Moreover, because of the linearity of the Stokes equations, the resulting parallelization is truly embarassing (in the computational sense, not in the time it took me to realize this property), consisting of $N_{k}^{2}$ independent tasks (for $N_{k}$-by- $N_{k}$ Fourier truncations of the interactions) which can be distributed by the simulation process onto separate computational engines by way of passing the current particle positions and limited pre-computed information common to all tasks. 

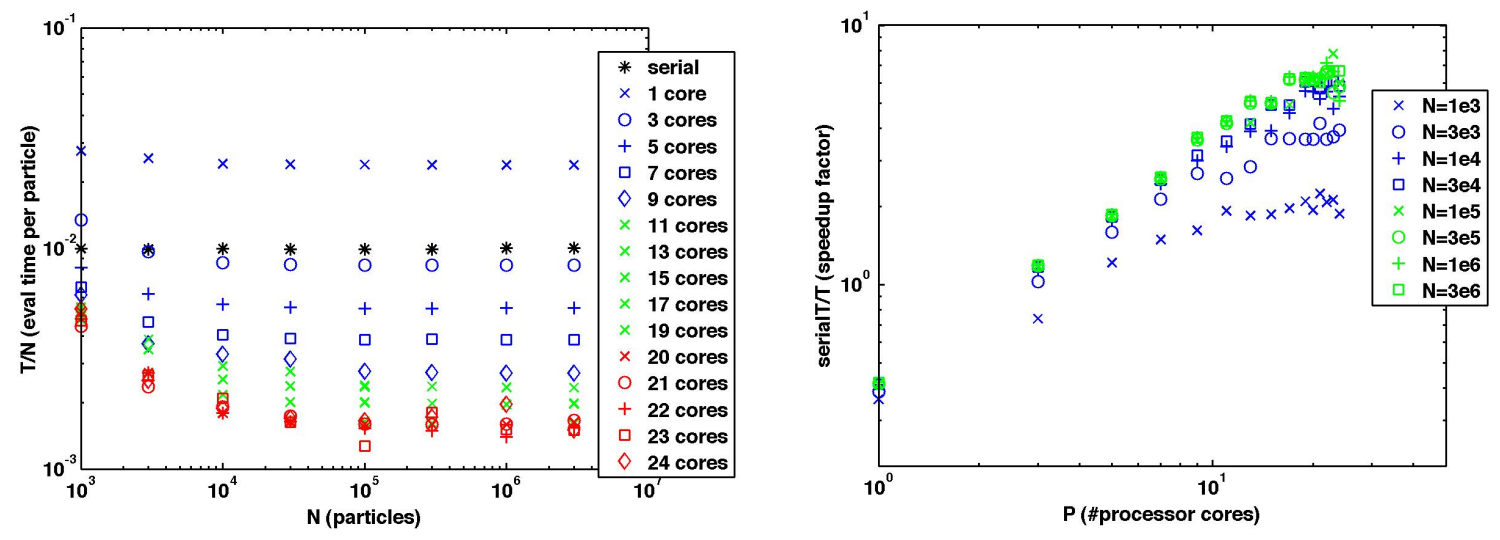

Figure 3: Simple test timings of the wavevector-space parallelization scheme, evaluating the particle velocities for a single set of randomly-selected particle positions on a $6 \mathrm{x}$-quad-core TyanPSC Typhoon cluster, with parallelization into $N_{k}=63$ line segments in the 2-D wavevector space. (a) Evaluation time per particle v. number of particles $N$, for different numbers of processor cores. (b) The speedup factor compared to the serial-code calculation v. number of processor cores $P$, for different numbers of particles.

We present here a relatively crude example of the resulting speedup, using a TyanPSC Typhoon series cluster consisting of 6 quad-core Intel Xeon X5355 processors (purchased with Mucha's UNC startup package). In Figure 3, we compare computational times taken to calculate the velocities for a single collection of $N$ randomly-positioned particles. We note that the original serial code is, strictly speaking, $O(N \ln N)$ because of the required sorting (quick-sort) of particles along the coordinate perpendicular to the walls. Practically speaking, however, the computational cost has been observed to scale only as $O(N)$ (not demonstrated here), even for $N$ as large as $3 \cdot 10^{6}$, because even at this large $N$ the sort is relatively fast compared to the $O(N)$ bulk of the calculation. In the present test, the calculation was parallelized into $N_{k}=63$ independent tasks (each corresponding to an $N_{k}$-long line segment in wavevector space). The relatively coarse granularity of these tasks relative to the number of processor cores certainly affects the plotted results, with most of the tests peaking in speedup with 21 processor cores enabled ( 3 tasks per processor core).

We also note the $\approx 3 \times$ overhead cost of implementing the single-core parallelization on this cluster as compared to the original serial code, as evidenced in Figure 3; but we remark that this test was performed on an incompletely-configured cluster without the full Infiniband enabled. Similar tests on dual-core and quad-core desktop machines have demonstrated an overhead factor of only $\approx 1.4$, and we expect similar performance on the 24-core cluster once it is fully configured. Even with the large overhead here, however, we see that this scheme on the presently-configured cluster provides order-of-magnitude speedup compared to using the serial code on a single processor. While most applications that continue to require consideration of multiple realizations will hence be simulated faster by multiple serial instances, the ability to rapidly generate even larger simulations will prove useful in the future.

The DOE award will be cited in any future publications or presentations resulting from the use of this parallel version of the code. 


\section{Final Thoughts}

Approximately $70 \%$ of the budget of this award was used to support student research, including stipends, tuition, and overhead, with approximately 72 person support months in the award (plus PI summer salary). It is thus important to stress the direct contributions made both by this award toward supporting students and by the students toward the research aims of the proposal. Students supported by this award have included GT graduate students Svetlana Bukharina, Christel Hohenegger, Nipun Kwatra, Vidit Nanda, Chris Wojtan, and UNC graduate student Swathi Guda. Of these, Christel Hohenegger was far and away the dominant recipient of support from this DOE award, and one of the main contributors to results obtained under this award. It is with no small amount of pride that I inform you that your student support was primarily spent supporting Dr. Hohenegger, who is currently a postdoctoral associate at the Courant Institute at NYU.

I would also like to say that I am personally very grateful to the ECPI in Applied Mathematics program. As a practical matter, I am very thankful for the help provided by the program managers and administrators who helped me get this award transferred properly and repeatedly extended after my Summer 2005 move to UNC. More generally, this award from the DOE fabulously enriched my years as a tenure-track assistant professor, first at GT and then at UNC, providing me both with important recognition and with much appreciated support to build on my previous research studies and to take those results and techniques in new scientific directions.

If you have any additional questions about any of the activities performed or results obtained under this award, please do not hesitate to contact me.

\section{RefERENCES Cited}

[1] N. Abaid, D. Adalsteinsson, A. Agyapong, and R. M. Mclaughlin, An internal splash: Levitation of falling spheres in stratified fluids, Phys. Fluids, 16 (2004), pp. 1567-1580.

[2] M. Carlson, P. J. Mucha, and G. Turk, Rigid Fluid: Animating the interplay between rigid bodies and fluid, ACM Trans. Graph. (SIGGRAPH), 23 (2004), pp. 377-384.

[3] I. C. Carpen And J. F. Brady, Gravitational instability in suspension flow, J. Fluid Mech., 472 (2002), pp. 201-210.

[4] F. Graf, E. Meiburg, and C. HÄrtel, Density-driven instabilities of miscible fluids in a heleshaw cell: linear stability analysis of the three-dimensional stokes equations, J. Fluid Mech., 451 (2002), pp. 261-282.

[5] P. J. Mucha And M. P. Brenner, Diffusivities and front propagation in sedimentation, Phys. Fluids, 15 (2003), pp. 1305-1313.

[6] N. A. Patankar, P. Singh, D. D. Joseph, R. Glowinski, and T. W. Pan, A new formulation of the distributed Lagrange multiplier/fictitious domain method for particulate flows, Int. J. Multiphase Flow, 26 (2000), pp. 1509-1524.

[7] R. SAdR, H. LI, AND M. YodA, Impact of hindered Brownian diffusion on the accuracy of nano-particle image velocimetry, submitted to Exp. in Fluids, (2004).

[8] R. Sadr, M. Yoda, Z. Zheng, and A. T. Conlisk, An experimental study of electroosmotic flow in rectangular microchannels, J. Fluid Mech., 506 (2004), pp. 357-367. 